\title{
Error assessment of GOCE SGG data using along track interpolation
}

\author{
J. Bouman ${ }^{1}$ and R. Koop ${ }^{1}$ \\ ${ }^{1}$ SRON National Institute for Space Research, Utrecht, The Netherlands
}

\begin{abstract}
GOCE will be the first satellite gravity mission measuring gravity gradients in space using a dedicated instrument called a gradiometer. High resolution gravity field recovery will be possible from these gradients. Such a recovery requires a proper description of the gravity gradient errors, where the a priori error model is for example based on end-to-end instrument simulations. One way to test the error model against real data, i.e. to see if the a priori model really describes the actual error, is to compare along track interpolated gradients with the measured gradients. The difference between the interpolated and measured gravity gradients is caused by, among others, the interpolation error and the measurement errors. The idea is that if the interpolation error is small enough, then the differences should be predicted reasonably well by the error model. This paper discusses a simulation study where the gravity gradient errors are generated with an end-to-end instrument simulator. The measurement error will be compared with the interpolation error and we will assess the latter as a function of the sampling interval.
\end{abstract}

\section{Introduction}

The main goal of the GOCE mission (expected to be launched early 2006) is to provide unique models of the Earth's gravity field and the geoid, on a global scale with high spatial resolution and to very high accuracy (ESA, 1999). To this end, GOCE will be equipped with a GPS receiver for high-low satellite-to-satellite tracking (SST-hl) observations, and with a gradiometer for observation of the gravity gradients (SGG). The gradiometer consists of six 3-axes accelerometers mounted in pairs along three orthogonal arms. From the readings of each pair of accelerometers the socalled common mode (CM) and differential mode (DM) signals are derived. The CM observations are used to obtain information about the linear accelerations and are input to the drag free control system. The measurements of the $\mathrm{CM}$ are

Correspondence to: J. Bouman (J.Bouman@sron.nl) also needed for accurate separation of the non-conservative and conservative forces and are therefore important for the long wavelength gravity field recovery from SST measurements. The DM observations are used to derive the required gravity gradients. The accelerometers and the gradiometer are designed such as to give the highest achievable precision in the measurement bandwidth (MBW) between 5 and 100 $\mathrm{mHz}$. For the diagonal gravity gradients in a Local Orbital Reference Frame (LORF, $X$-axis in the velocity direction, the $Z$-axis approximately radially outward and the $Y$-axis complements the right-handed frame) this precision will not exceed $4 \mathrm{mE} / \sqrt{\mathrm{Hz}}\left(1 \mathrm{E}=10^{-9} \mathrm{~s}^{-2}\right)$ in the MBW (Cesare, 2002).

The observations will be contaminated with stochastic and systematic errors. For the GOCE gradiometer, systematic errors typically are due to instrument imperfections like misalignments of the accelerometers, scale factor mismatches etc. The CM and DM couplings, which are the result of such instrument imperfections, can be determined to an accuracy level of $10^{-2}-10^{-4}$ prior to the mission by the so-called pre-flight calibration on ground using a test bench (Cesare, 2002). In orbit, a so-called internal calibration procedure will be used (ESA, 1999), by which the CM and DM couplings can be determined to an accuracy level at which their effect on the gradients in the MBW stays below the required $4 \mathrm{mE} / \sqrt{\mathrm{Hz}}$. The values of the calibration parameters (elements of the calibration matrix) are measured by putting a known acceleration signal on the gradiometer in orbit using the thrusters. After this procedure, the CM and DM read-outs of the gradiometer are corrected using the measured calibration parameters.

The gravity gradients are derived from the internally calibrated DM accelerations. The internal calibration, however, is not sensitive to all instrument imperfections. For example, the true locations of the six accelerometers may differ from their nominal positions. This accelerometer mis-positioning as well as the read-out bias, for example, can not be accounted for. Therefore, in order to possibly correct for remaining errors after internal calibration, a third calibration 
step is required, which is called external calibration (or absolute calibration). It is performed during or after the mission and typically makes use of external gravity data (Arabelos and Tscherning, 1998; Koop et al., 2002).

Along with the external calibration of the observations, their error needs to be assessed. For this purpose, we could use either external data, such as terrestrial gravity data, or use the GOCE data themselves and perform an internal assessment. In view of the very high accuracy of GOCE in the MBW, it will be difficult to assess the gravity gradient errors in the MBW with the former method. We therefore focus on internal error assessment. Specifically, the pros and cons of along track data interpolation are studied. Albertella et al. (2000b) use along track interpolation for outlier detection, while the use of cross-overs and repeat tracks for error assessment is studied by Albertella et al. (2000a) and Koop et al. (2002), respectively.

The along track interpolation and error assessment is introduced in Sect. 2. The interpolation error is discussed in Sect. 3 for several cases, while the error assessment itself is discussed in Sect. 4.

\section{Along track interpolation}

GOCE will deliver time series of gravity gradients along its orbit. A subset of the time series may be used to interpolate at time $t=i$. The difference between the interpolated $\hat{y}(i)$ and the measured $y(i)$ is due to, among others, the interpolation error and the measurement errors. If the interpolation error is small enough then the above differences could be used for error assessment. The general interpolation model as well as the error assessment are discussed in more detail in this section.

\subsection{Model of condition equations}

The model of condition equations is

$\mathbf{B}^{T} E\{\underline{\boldsymbol{y}}\}=0 ; \quad D\{\underline{\boldsymbol{y}}\}=\mathbf{Q}_{\mathbf{y}}$

with least squares errors

$\underline{\hat{e}}=\mathbf{Q}_{\mathbf{y}} \mathbf{B}\left(\mathbf{B}^{T} \mathbf{Q}_{\mathbf{y}} \mathbf{B}\right)^{-1} \mathbf{B}^{T} \underline{\boldsymbol{y}}$.

The observations $\boldsymbol{y}$ are the gravity gradients $T_{X X}, T_{Y Y}, T_{Z Z}$ in the LORF. The anomalous gravity gradients $T_{j j}$, with $j=X, Y, Z$, are obtained by taking the difference between the measured gradients and some reference model values. The model $\mathbf{B}^{T}$ depends on the specific interpolation method, but for local interpolation methods $\mathbf{B}^{T}$ will be sparse. The central idea is: interpolate one or more observations from other observations and compare the interpolated values with the original ones. The error variance-covariance matrix $\mathbf{Q}_{\mathbf{y}}$ is full in general. Due to the coloured noise behaviour of the gradiometer, the gravity gradient errors will be correlated in time.

\subsection{Overall model test}

An unbiased estimate of the variance of unit weight is

$\hat{\sigma}^{2}=\frac{\hat{\boldsymbol{e}}^{T} \mathbf{Q}_{\mathbf{y}}^{-1} \hat{\underline{\boldsymbol{e}}}}{b}$

with $b$ the rank of $\mathbf{B}$, that is, the number of independent condition equations. Thus $\hat{\sigma}^{2}$ depends on the a priori error matrix $\mathbf{Q}_{\mathbf{y}}$ and the a posteriori error $\underline{\hat{\boldsymbol{e}}}$. In practice one could use Eq. (3) to test whether one a priori error model is to be preferred over the other. The closer $\underline{\sigma}^{2}$ is to 1 , the more likely it is that the corresponding a priori model $\mathbf{Q}_{\mathbf{y}}$ describes the errors well assuming that outliers have been removed and that the variance of unit weight is 1 .

The distribution of $\hat{\sigma}^{2}$ under the null hypothesis $H_{0}$ (i.e. the a priori error model is correct) is given as

$H_{0}: \underline{\hat{\sigma}}^{2} \sim F(b, \infty, 0)$

that is a central $F$ distribution. The a priori error model will be rejected if $\hat{\sigma}^{2}>k_{\alpha}$, that is, if the a posteriori variance of unit weight is larger than a critical value with significance level $\alpha$. For example $F_{0.05}(60, \infty, 0)=1.4$, which means that for 60 conditions equations the a priori error model will be rejected if the a posteriori variance of unit weight is larger than 1.4. In approximately $5 \%$ of the cases the null hypothesis will be rejected although the a priori error model is correct.

\subsection{Comparison with other methods}

The advantage of along track interpolation for error assessment is that it can be started right after the first measurements are available (provided that the on board navigation solution is accurate enough). There are no conditions on the repeat of the orbit or on the cross-overs. Interpolation methods typically use local data, which means that this method may be suited to assess the error especially for high frequencies, that is, near or inside the MBW. A disadvantage may be that systematic, long wavelength errors will not be visible using local interpolation methods. Hence, long wavelength errors could go undetected. This problem may be overcome using global interpolation methods, however, this may increase the interpolation error.

Koop et al. (2002) discuss the use of repeat tracks for internal error assessment, while Albertella et al. (2000a) use cross-overs. Both methods are in the form of model (1). Cross-overs are not suited to test the $T_{X X}$ and $T_{Y Y}$ errors due to large measurement errors in the off-diagonal gravity gradients, which are needed to transform the ascending and descending measurements to a common frame. The radial gravity gradient $T_{Z Z}$, however, can be tested. Especially, the cross-overs after one or a few revolutions can be used, while the cross-overs further apart in time are less useful. The radial orbit variations at cross-overs tend to increase in time, which affects the comparisons (Albertella et al., 2000a). In addition, cross-overs are less dense than the along track sampling, so the high frequency errors can not be assessed. 


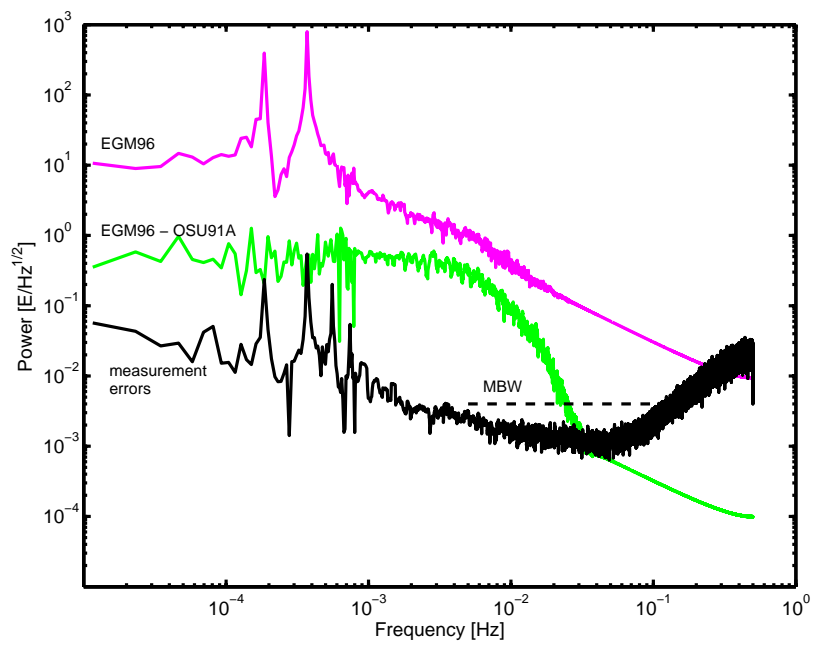

Fig. 1. Spectral densities of the $T_{Y Y}$ signal and measurement errors.

In principle, repeat tracks can be used to test the whole spectral range, which is a clear advantage. The method, however, heavily relies on a close repeat of the orbit after some time. Specifically the radial distance, that is, the distance in the $Z$-direction, between repeat tracks has to be small, and therefore Koop et al. (2002) consider a so-called frozen orbit, which minimizes the radial orbit variations. It is, however, unlikely that the GOCE orbit will be a frozen orbit and a repeat is even avoided as much as possible within two months to get a dense spatial cross track coverage, (see ESA, 1999).

These three internal error assessment methods (along track interpolation, cross-overs, repeat tracks) are therefore more or less complementary.

\section{Interpolation error}

The along track interpolation errors should be small enough compared to the measurement errors. This is a necessary condition for the along track interpolation method to be useful. Two simple interpolation methods are tested: linear interpolation and Overhauser splines (Overhauser, 1968). Although the interpolation methods do not require so, it is assumed for simplicity that the along track sampling is regular. In practice this will probably be realized for many time intervals during the GOCE mission and it is certainly true for our simulations. Results for linear interpolation will not be shown. Despite the interplation error is small compared to the measurement error for this method, Overhauser splines yield a factor of two smaller interpolation errors. The former method will therefore not be used in this paper.

True gravity gradients in the LORF were generated using the global gravity field model EGM96. Anomalous gravity gradients were computed by subtracting reference gravity gradients computed with OSU91A. These anomalous gravity gradients will be used in the interpolation error test. First, no errors are added to the measurements and the orbit is assumed to be known. Then the residuals $\mathbf{B}^{T} \boldsymbol{y} \neq 0$ are entirely
Table 1. RMS of gravity gradient measurement errors and Overhauser spline interpolation errors in $[\mathrm{mE}]$

\begin{tabular}{lccc}
\hline & $T_{X X}$ & $T_{Y Y}$ & $T_{Z Z}$ \\
\hline interpolation errors & 0.04 & 0.01 & 0.04 \\
measurement errors & 8.2 & 9.7 & 8.5 \\
\hline
\end{tabular}

due to the interpolation. The gravity gradient data sets have a length of 1 day and the sampling interval is $1 \mathrm{~s}$.

Overhauser splines are cubic splines that are one time continuously differentiable at the data points (Overhauser, 1968). For equidistant data points the condition equations take the form

$$
\begin{array}{r}
y(i)=\frac{7}{12}[y(i-k)+y(i+k)]+ \\
-\frac{1}{12}[y(i-2 k)+y(i+2 k)]
\end{array}
$$

where $k=1$ or 2 or .... If $k=1$ then neighbouring points are used for the interpolation, if $k=2$ then every second point is used, etc. Suppose $k=1$ and there are $m$ measurements, then $i$ may take the values $3, \ldots, m-2$.

Table 1 lists the Root Mean Square (RMS) of the gravity gradient measurement errors as well as the RMS of interpolation errors due to interpolation with Overhauser splines. The former errors are obtained after external calibration of the original gravity gradients as described in Koop et al. (2002). The errors due to interpolation are therefore two to three orders smaller than the measurement errors, with the $T_{X X}$ and $T_{Z Z}$ interpolation errors larger than the $T_{Y Y}$ errors.

The spectral densities of the full and residual signal as well as the measurement errors are shown, for $T_{Y Y}$, in Fig. 1, whereas the interpolation errors are shown in Fig. 2. The error due to interpolation is below the measurement error over the entire spectrum. Since the interpolation is local, the interpolation error is small for the low frequencies. In the MBW the interpolation error is clearly correlated with the residual signal, that is, with the gravity gradient anomalies (EGM96 - OSU91A). It should be noted that degree 360 is the maximum spherical harmonic degree of the gravity field models in the simulations. At the Earth's surface this corresponds to a resolution of roughly $55 \mathrm{~km}$, which is a distance the GOCE satellite will travel in $7 \mathrm{~s}$, given its along track velocity of $7.8 \mathrm{~km} / \mathrm{s}$. Consequently, the true gravity signal is probably not well presented in our simulations for frequencies larger than $7 \times 10^{-2} \mathrm{~Hz}$ or so. Above the MBW, $>0.1 \mathrm{~Hz}$, the interpolation error increases. Since there is practically no residual signal in the current simulation above the MBW, this may be caused by round-off errors. The small peak at $0.1 \mathrm{~Hz}$ is not well understood. The interpolation error in the MBW is slightly larger for $T_{X X}$ and $T_{Z Z}$, although still well below the measurement error.

In general the interpolation error increases for increasing sample distance. This is shown in Fig. 2 for the time series 


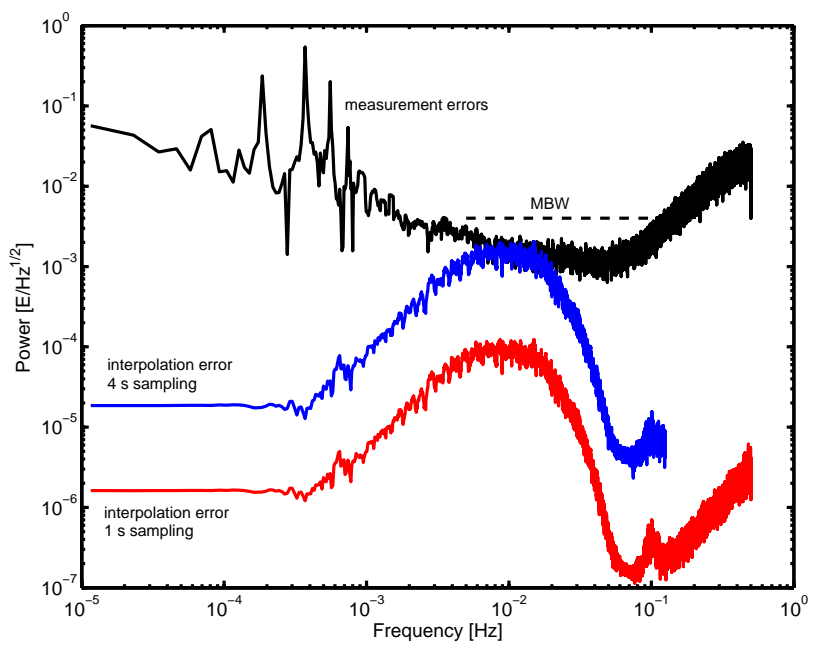

Fig. 2. Spectral densities of measurement and interpolation errors for $T_{Y Y}$.

resampled such that time distance between successive observations is $4 \mathrm{~s}$. The interpolation error is smaller than the measurement error for samples up to $2 \mathrm{~s}$ distance for all frequencies (not shown). The interpolation error is larger than the measurement error in the MBW for $T_{X X}$ and $T_{Z Z}$ for a sample distance of $4 \mathrm{~s}$, whereas the interpolation error for $T_{Y Y}$ is still below the measurement error for all frequencies at this sampling interval, as is shown in Fig. 2.

So far, overlapping intervals have been used, that is, observations $1,2,4$, and 5 are used to predict 3 , then $2,3,5$, and 6 are used to predict 4 , etc. One could also use nonoverlapping intervals, that is, use observations $1,2,4,5$ to predict 3 , then use $6,7,9,10$ to predict 8 , etc. Since the interpolation error is a systematic error (it depends on the signal), the error decreases slightly for the non-overlapping case: there are 5 times less condition equations (results are not shown).

\section{Statistical test results}

In the previous section it was shown that for a $1 \mathrm{~s}$ sample interval the interpolation error can be neglected compared to the measurement error. The model of condition Eqs. (1) holds, and the appearance of $\mathbf{B}^{T}$ depends on whether we use $1 \mathrm{~s}$ shifts or $2 \mathrm{~s}$ shifts etc. between successive condition equations. The error assessment requires that an a priori error model $\mathbf{Q}_{\mathbf{y}}$ is available. Our first aim is therefore to compute such an error model, and to study its characteristics.

In general, the error variance-covariance matrix is full. The errors on the GOCE gravity gradients are coloured, that is, the error is small in the MBW and increases outside the MBW, see also Fig. 1. This yields along track error correlation. If the full data period is considered ( 1 day) then error correlations exist that can not be neglected. Especially for $T_{Y Y}$ and $T_{Z Z}$ this is true because 1,2, etc. cpr (cycles per revolution) errors are present, see Figs. 2 and 3. These errors
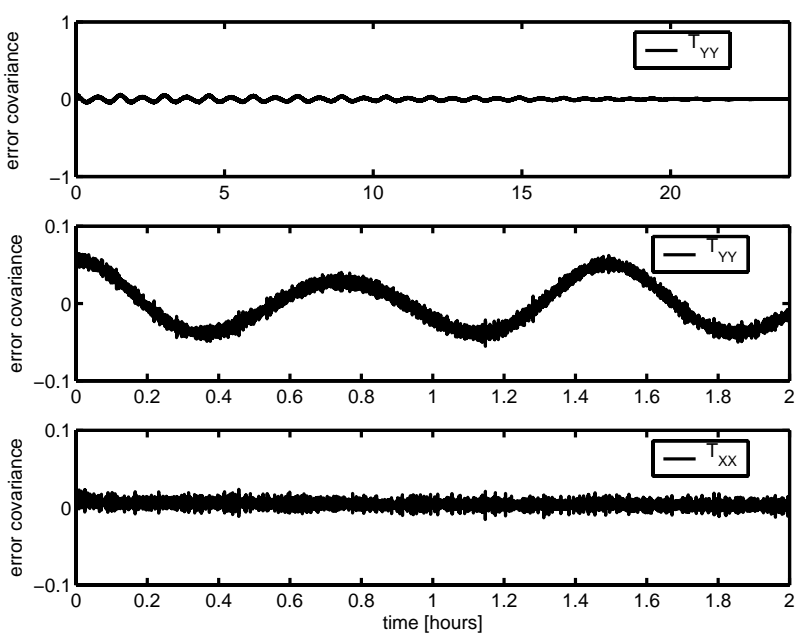

Fig. 3. Gravity gradient error covariances for 1 day. Top panel: $T_{Y Y}$ 1 day; middle panel: $T_{Y Y}$ zoom in on first $2 \mathrm{~h}$; bottom panel: $T_{X X}$ zoom in on first $2 \mathrm{~h}$.

are much smaller for $T_{X X}$, which results in much smaller error correlations. Shown in the top panel of Fig. 3 are the true $T_{Y Y}$ error covariances for the total time series of 1 day, scaled with the variance at $t=0$. Hence, at $t=0$ the error covariance is 1 , but this is hard to distinguish in the present graph. Zooming in on the first $2 \mathrm{~h}$ of the error covariances shows strong correlations (middle panel). The error covariances of $T_{Z Z}$ are similar to those of $T_{Y Y}$. Because external calibration significantly reduced the $T_{X X}$ errors at 1 and $2 \mathrm{cpr}$, the correlation is less for longer time intervals (see Fig. 3, bottom panel).

The overall model test requires the inversion of the (full) error matrix $\mathbf{Q}_{\mathbf{y}}$, Eq. (3). Limiting our test to time windows of $1000 \mathrm{~s}$, for example, would allow straightforward computations. A disadvantage is that the test of the validity of a priori error models becomes limited. However, the most interesting part of GOCE, the MBW, is contained in these windows and the along track interpolation, which we study here, is not sensitive to longer wavelengths. Figure 4 shows the $T_{Y Y}$ error covariances, averaged over 86 windows of $1000 \mathrm{~s}$. Before the error covariance of each window is computed, the mean was subtracted, which in effect removes part of the long wavelength errors. Therefore, the average error covariance for the full $1000 \mathrm{~s}$ period appears to be noisy (top panel). Zooming in, however, on the first $100 \mathrm{~s}$ shows that correlations up to $10-15 \%$ still remain (Fig. 4, middle panel). The correlation is strongest for the first 2-3 s (bottom panel). The $T_{X X}$ and $T_{Z Z}$ error covariances for $1000 \mathrm{~s}$ show similar behaviour to those of $T_{Y Y}$.

The averaged empirical error covariance functions for the $1000 \mathrm{~s}$ windows have been used to create three a priori error matrices $\mathbf{Q}_{\mathbf{y}}$ (one for each studied gravity gradient). Since these matrices are based on the true (simulated) errors, one should expect that the a priori error model will be accepted for nearly all error assessment windows. Two ex- 

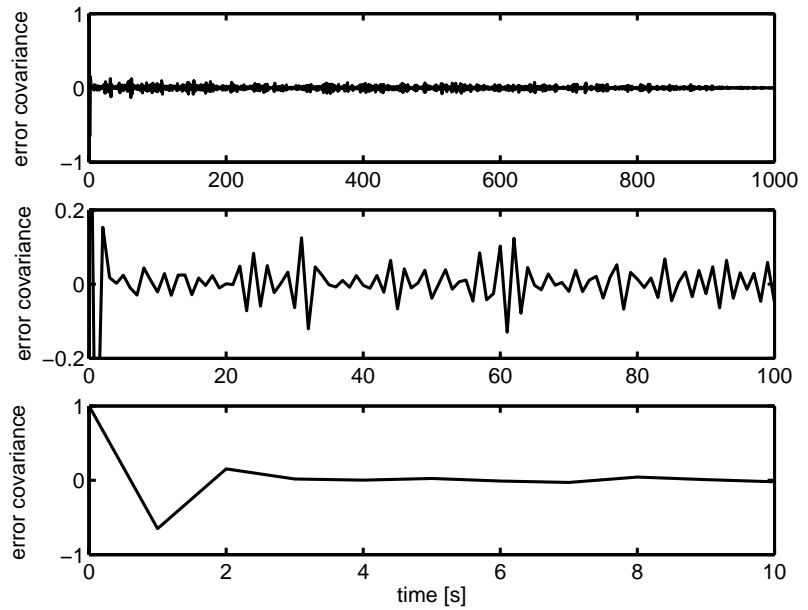

Fig. 4. $T_{Y Y}$ error covariances averaged over $1000 \mathrm{~s}$ windows. Top panel: full $1000 \mathrm{~s}$ period; middle panel: zoom in on first $100 \mathrm{~s}$; bottom panel: zoom in on first $10 \mathrm{~s}$.

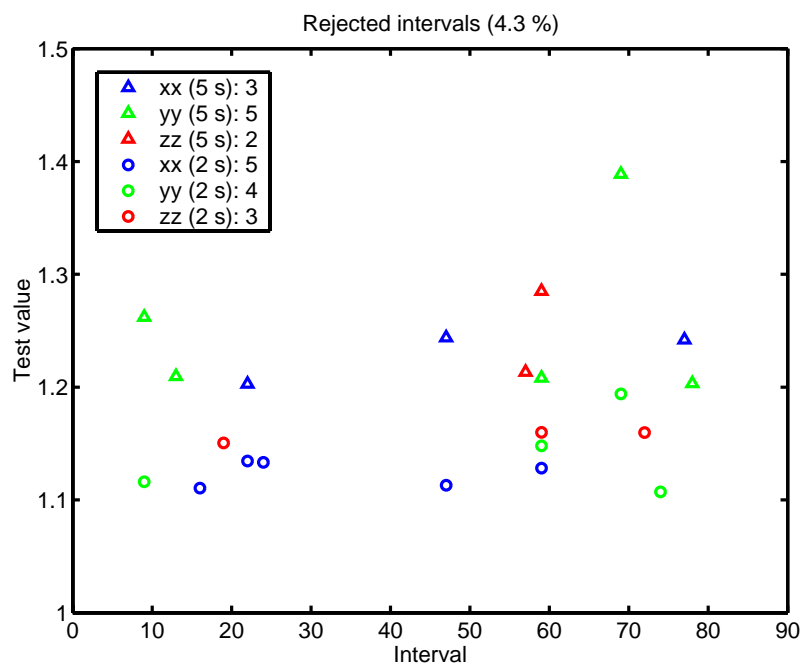

Fig. 5. Rejected $\underline{\hat{\sigma}}^{2}$ values. In total 22 out of $6 * 86=516$ values are rejected.

amples are shown in Fig. 5 and Tables 2 and 3. The triangles denote the rejected test values for interpolation in windows of $1000 \mathrm{~s}$ length, $1 \mathrm{~s}$ sampling and $5 \mathrm{~s}$ shifts between condition equations. Hence there are roughly 200 condition equations: $F_{0.05}(200, \infty, 0) \approx 1.2$. The circles denote the rejected test values for interpolation in windows of $1000 \mathrm{~s}$ length, $1 \mathrm{~s}$ sampling and $2 \mathrm{~s}$ shifts between condition equations. Hence there are roughly 500 condition equations: $F_{0.05}(500, \infty, 0) \approx 1.1$. In total $4.3 \%$ of the a posteriori variances are too large and rejected, which is a satisfactory result.

As the bottom panel of Fig. 4 suggests, the full error matrix $\mathbf{Q}_{\mathbf{y}}$ can be approximated by a sparse matrix (band matrix). Considering the error covariance functions, we use the first 4 points of the covariance functions. The number of rejected a
Table 2. Average a posteriori variance of unit weight and $F$-test results. Error assessment window is $1000 \mathrm{~s}$ (86 windows), sampling interval is $1 \mathrm{~s}$, interpolation shift is $2 \mathrm{~s}$

\begin{tabular}{ccccccccc}
\hline \multirow{2}{*}{$\mathbf{Q}_{\mathbf{y}}$} & \multicolumn{2}{c}{ full } & \multicolumn{2}{c}{ band } & \multicolumn{2}{c}{ diag } & \multicolumn{2}{c}{$\mathbf{Q}_{\mathbf{y}}$ of $T_{X X}$} \\
\cline { 2 - 9 } & $\underline{\hat{\sigma}}^{2}$ & rej. & $\underline{\hat{\sigma}}^{2}$ & rej. & $\underline{\hat{\sigma}}^{2}$ & rej. & $\underline{\hat{\sigma}}^{2}$ & rej. \\
\hline$T_{X X}$ & 1.00 & 5 & 1.00 & 5 & 1.80 & 86 & 1.00 & 5 \\
$T_{Y Y}$ & 1.00 & 4 & 1.00 & 5 & 1.76 & 86 & 1.35 & 86 \\
$T_{Z Z}$ & 1.00 & 3 & 1.00 & 5 & 1.74 & 86 & 1.00 & 5 \\
\hline
\end{tabular}

Table 3. Average a posteriori variance of unit weight and $F$-test results. Error assessment window is $1000 \mathrm{~s}$ (86 windows), sampling interval is $1 \mathrm{~s}$, interpolation shift is $5 \mathrm{~s}$

\begin{tabular}{ccccccc}
\hline \multirow{2}{*}{$\mathbf{Q}_{\mathbf{y}}$} & \multicolumn{2}{c}{ full } & \multicolumn{2}{c}{ band } & \multicolumn{2}{c}{$\mathbf{Q}_{\mathbf{y}}$ of $T_{X X}$} \\
\cline { 2 - 7 } & $\hat{\underline{\sigma}}^{2}$ & rej. & $\hat{\underline{\sigma}}^{2}$ & rej. & $\hat{\underline{\sigma}}^{2}$ & rej. \\
\hline$T_{X X}$ & 0.99 & 3 & 1.00 & 3 & 0.99 & 3 \\
$T_{Y Y}$ & 1.00 & 5 & 1.01 & 4 & 1.35 & 77 \\
$T_{Z Z}$ & 1.01 & 2 & 1.01 & 2 & 1.01 & 3 \\
\hline
\end{tabular}

posteriori variances increases slightly: $4.7 \%$ is rejected. $\mathrm{Ne}$ glecting all correlations and using the variances only does not give satisfactory results. The a priori error model is rejected for all error assessment windows. See Tables 2 and 3.

If the a priori error matrix $\mathbf{Q}_{\mathbf{y}}$ of $T_{X X}$ is used for the other two gradients, then it is rejected for (nearly) all $T_{Y Y}$ windows, whereas the $T_{Z Z}$ results stay the same, see Tables 2 and 3 . This is a consequence of the fact that, in our simulation for short time intervals, the $T_{X X}$ error covariances are almost equal to those of $T_{Z Z}$. The differences with the $T_{Y Y}$ error are somewhat larger for short time intervals. The error assessment using along track interpolation is therefore rather sensitive to the correct error model. Or at least, sensitive to too optimistic error models. If we use a pessimistic error model, for example multiply the error model of $T_{Z Z}$ with a factor of 1.5 , then the a priori error model is accepted for all windows. However, the average a posteriori variance of unit weight is $\hat{\sigma}^{2}=1 / 1.5$ for 86 windows. It may therefore be necessary to apply a double-sided statistical test instead of one-sided test as we do now.

Finally, a $1 \mathrm{~s}$ sampling and $1 \mathrm{~s}$ condition equation shift yields a badly conditioned $\mathbf{B}^{T} \mathbf{Q}_{\mathbf{y}} \mathbf{B}$ matrix. Consequently, the test results are unreliable. This may be a consequence of too much correlation or may have some other cause, yet unresolved.

\section{Conclusions and discussion}

Along track interpolation is well suited to test the a priori error model for GOCE SGG observations. This method is complementary to other methods of error assessment such as the use of repeat tracks and cross-overs. The major advantage 
of along track interpolation is that it is independent of the repeat conditions of the satellite ground track and that it may be applied real time. Thus, in the context of quick-look data quality assessment, this tool is a major candidate for real time monitoring of the instrument in flight.

The assumptions underlying the current simulation study are that no outliers are present and that the orbit navigation solution is accurate enough. In reality, outliers will be present, and they have to be dealt with before the interpolation error assessment is performed. Outliers can be removed on basis of an a priori error model. Hence, a kind of iterative procedure comes to mind. Further, orbit uncertainties in the navigation solution contribute to the interpolation error, since the method assumes known locations of the measurements, and the observations are reduced w.r.t. an a priori gravity model. It has to be assessed what the upper limit is for the accuracy of the navigation solution for our method to work properly.

The interpolation error is very small compared to measurement error for $1 \mathrm{~s}$ sampling. For the $T_{X X}$ and $T_{Z Z}$ gradients the interpolation error is larger than for the $T_{Y Y}$ gradient. In our simulations we used a reference model and subtracted it from the simulated gravity gradients, which gives residual gravity gradients. Since the maximum spherical harmonic degree is 360 in these simulations, there is concern that the residual signal is unrealistic for high frequencies. Or, in other words, the residual gravity gradient signal may be much larger for high frequencies when reference gravity gradients are subtracted from true, measured gradients. How this influences the interpolation needs to be studied.

Error assessment windows are limited to $1000 \mathrm{~s}$ or so due to computer restrictions. An advantage is that the error correlation is largest for the first few seconds, so that a priori error matrix $\mathbf{Q}_{\mathbf{y}}$ may be approximated by a band matrix. In addition, the interpolation methods are local and therefore probably not too sensitive to long wavelength errors. Disadvantage is that not the entire spectrum can be tested. Importantly, the entire GOCE MBW is contained in our test.

We accept the a priori error model if the $F$-test does not reject too many a posteriori variances of unit weight (based on a chosen signifigance level) and the average a posteriori variance of unit weight has to be "close" to one. In this way, we can use this method in reality to test any choice of an a priori error model to find the best model for the real observations. In order to prevent a false acceptance of a too pessimistic a priori model, our one-sided statistical test should be replaced by a double-sided test.

If the a priori error model is rejected then either the model is wrong indeed or the interpolation error is too large. We may then increase the sample interval, a factor of two for example. If the statisical test yields the same results then the a priori model is false. If more points are rejected then at least the interpolation error in the latter test is too large. We can not say more about the former test. We therefore need information on the interpolation error beforehand.

Acknowledgements. The satellite orbit and the EGM96 gravity gradients were provided by Karl Heinz Ilk as part of simulation scenarios of Special Commission 7 in cooperation with Special Study Group 2.193 of the International Association of Geodesy, which is appreciated. Volker Hannen computed the simulated gravity gradient errors, which is appreciated as well. We also acknowledge the use of newmat, a C++ matrix library developed by Robert Davies. The software can be downloaded from the Internet, and this matrix library supported part of the computations.

\section{References}

Albertella, A., Migliaccio, F., Sansò, F., and Tscherning, C.: The space-wise approach - overall scientific data strategy, in: From Eötvös to mGal, Final Report, (Ed) Sünkel, H., ESA/ESTEC contract no. 13392/98/NL/GD, 2000a.

Albertella, A., Migliaccio, F., Sansò, F., and Tscherning, C.: Scientific data production quality assessment using local space-wise pre-processing, in: From Eötvös to $\mathrm{mGal}$, Final Report, (Ed) Sünkel, H., ESA/ESTEC contract no. 13392/98/NL/GD, 2000b.

Arabelos, D. and Tscherning, C.: Calibration of satellite gradiometer data aided by ground gravity data, Journal of Geodesy, 72, 617-625, 1998.

Cesare, S.: Performance requirements and budgets for the gradiometric mission, Issue 2 GO-TN-AI-0027, Preliminary Design Review, Alenia, 2002.

ESA: Gravity Field and Steady-State Ocean Circulation Mission, Reports for mission selection; the four candidate earth explorer core missions, ESA SP-1233(1), 1999.

Koop, R., Bouman, J., Schrama, E., and Visser, P.: Calibration and error assessment of GOCE data, in: Vistas for Geodesy in the New Millenium, (Eds) Ádám, J. and Schwarz, K.-P., vol. 125 of International Association of Geodesy Symposia, pp. 167-174, Springer, 2002.

Overhauser, A.: Analytic definition of curves and surfaces by parabolic blending, Techn. Report No. SL68-40, Scientific Research Staff Publication, Ford Motor Company, Detroit, 1968. 